\title{
HESS-II reconstruction strategy and performance in the low-energy (20-150 GeV) domain
}

\author{
Y. Becherini*, A. Djannati-Ataï, M. Punch*, K. Bernlöhr ${ }^{\dagger}$, S. Ehlert ${ }^{\dagger}$, J. Masbou**, \\ E. Moulin ${ }^{*}$ and M. Paz Arribas ${ }^{\S, \pi}$ \\ ${ }^{*}$ APC, AstroParticule et Cosmologie, CNRS - Univ. Paris VII, 10, rue A. Domon et L. Duquet, 75205 Paris \\ Cedex 13, France \\ ${ }^{\dagger}$ Max-Planck Institut für Kernphysik, P.O. Box 103980, D-69029, Heidelberg, Germany \\ ** LAPP Laboratoire d'Annecy-le-vieux de Physique de Particules, Chemin de Bellevue, BP 110, 74941, \\ Annecy-le-vieux cedex, France \\ ${ }^{\ddagger}$ CEA/Saclay, IRFU/SPP, 91191 Gif-sur-Yvette, France \\ ${ }^{\S}$ Humboldt Universität zu Berlin, Newtonstr. 15, D-12489 Berlin, Germany \\ "DESY Zeuthen
}

\begin{abstract}
In mid-2009 a notable upgrade of the H.E.S.S. telescope system will take place: a new telescope with a $600 \mathrm{~m}^{2}$ mirror area and very-high-resolution camera $\left(0.07^{\circ}\right)$ will be positioned at the centre of the present configuration, with the aim of lowering the threshold and enhance its sensitivity in the $100 \mathrm{GeV}$ to several TeV energy range. HESS-II will permit the investigation of the lower energy $\gamma$-ray spectra in various cosmic accelerators, giving information on the origin of the $\gamma$-rays observed, and will detect AGNs with a redshift greater than 0.2 (being less affected by absorption by Extragalactic Background Light - EBL - in this energy range) and will search for new classes of very high energy $\gamma$-ray emitters (pulsars, microquasars, GRB, and dark matter candidates).
\end{abstract}

Keywords: Event reconstruction, angular resolution, energy resolution, effective area

PACS: <Replace this text with PACS numbers; choose from this list: http://www. aip..org/pacs/index.html>

\section{INTRODUCTION}

By the end of 2009 the H.E.S.S. experiment will enter its Phase II and at that time the data taking configuration will consist of the four current telescopes (here called T1, $\mathrm{T} 2, \mathrm{~T} 3$ and T4) plus the new very large HESS-II telescope (here called T5, see [1] at this conference for a detailed description of its structure). Since a new observational window is going to be opened in the range from $20 \mathrm{GeV}$ to about $100 \mathrm{GeV}$ with the largest ground-based Cerenkov telescope ever built, it is important to develop a powerful analysis in order to optimise the $\gamma$-ray detection in this energy range. We present here a first evaluation of the angular and energy resolution, as well as the effective detection area of this new configuration in the lowestenergy regime, focusing our attention on the pure mono T5 events. We have developed a shower reconstruction strategy for the events hitting the very large telescope alone. This consists of several consecutive steps: first of all we clean the images and evaluate the shower direction and core position (when possible) (see Sec. 3), then, knowing the basic parameters of the images and of the showers we can optimise and apply a cut for hadron rejection and a cut on the shape of the images (see Sec. 4), finally we estimate the event energy with the sample of selected events with a Neural Network (NN) approach (see Sec. 5).

\section{THREE-ENERGY DOMAIN ANALYSIS}

The intertelescope trigger system of the HESS-II configuration will operate in a hybrid mode, allowing to trigger on three classes of events in parallel: at very low energies on purely mono-telescope events of the very large telescope T5 (Mono domain), at mid energies on combined events with an image from the T5 and one from the smaller ones (T1 or T2 or T3 or T4) often with rudimentary information in the latter (semi-Stereo domain), and at even higher energies on current H.E.S.S. - Phase I type events with additional rich information in the central telescope (full-Stereo domain).

Monte Carlo Simulations and trigger conditions. For the evaluation of the low energy performance of the upgraded system we simulated gamma and proton showers between 20 and $150 \mathrm{GeV}$ at a zenith angle of $18^{\circ}$ assuming an optimal optical efficiency in the five telescopes. The $\gamma$-ray source is simulated on the optical axis, so the source is projected at the centre of the cameras, and the simulations are carried out over a sufficiently large radius $(500 \mathrm{~m})$ from the centre of the array. The local trig- 
TABLE 1. Local trigger parameters applied in the analysis presented in this paper for the four H.E.S.S. telescopes (T1, T2, T3, T4) and for the upcoming HESSII telescope (T5). An event is then accepted if it triggered at least one telescope.

\begin{tabular}{ccc}
\hline Parameter & T1, T2, T3, T4 & T5 \\
\hline pixel threshold (p.e.) & 2.5 & 3.5 \\
minimum number of pixels & 4 & 5 \\
\hline
\end{tabular}

ger configuration used in this analysis (see Tab. 1) can be summarised as follows: for $\mathrm{T} 1, \ldots, \mathrm{T} 4$ we required a pixel threshold of 2.5 p.e. and a minimum number of pixels of 4, while for T5 we raised these values to 3.5 and 5 to allow a better online rejection of low energy hadrons. The event is then kept only if it has at least one telescope satisfying the local trigger condition. The effective detection area at trigger level (see Fig. 3, upper continuous line) shows that up to about $90 \mathrm{GeV}$, events are mostly seen by the T5 telescope alone and that the detection maximum for the pure $\mathrm{T} 5$ events is reached at an energy of about $50 \mathrm{GeV}$. In the following analysis, we consider as pure Mono events all the showers triggering only the T5 telescope. As soon as even a little information is available from another telescope, the events are considered as stereo (since stereo reconstruction is more efficient).

\section{SHOWER RECONSTRUCTION}

Images are first of all cleaned with the following filtering rule: a pixel is accepted if it has at least a charge of 7 p.e. and some neighbouring pixels having a charge greater than 5 p.e. To reconstruct the shower parameters with the filtered pixels, we use the Hillas algorithm [2] which gives the moments of the images in each telescope. The Hillas parameters used are the length and the width of the major axis of a fitted ellipse, the angular distance between the centre of the camera and the barycentre of the image called nominal distance, the orientation angle between the fitted ellipse's axis and the source direction.

Stereo events. In the case of multi-telescope events, the direction and the core position are easily inferred by the intersection of the axes of the telescope images. For this kind of event, an additional reconstruction algorithm (called Model3D) is available, see [3]. The Model3D reconstruction algorithm is based on a 3-dimensional model of the shower in the atmosphere having the advantage of giving as a result some basic parameters of the air shower, as the length, the width, the altitude, and the number of photons generated by the shower.

Mono events. For pure Mono-telescope events, the shower direction has to be estimated from the measured

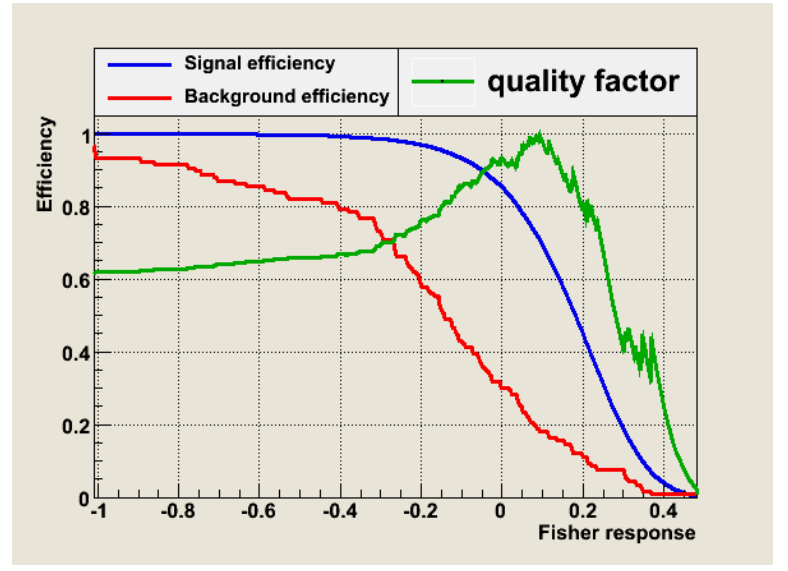

FIGURE 1. Signal S (upper line) and background B (lower line) efficiencies for the background rejection cut. The background rejection cut is obtained with a Fisher multi-variate analysis. The quality factor defined as $S / \sqrt{B}$ having a maximum at a Fisher response value of 0.1 is also shown.

parameters using relations derived from Monte Carlo simulations. The distance between the barycentre of the image and the source position along the shower major axis can be parametrized taking into account the image length and charge for example. What is not trivial to estimate in the Mono case is the shower core position: the same image could be given by a nearby low energy event or by a distant high energy event. The very large telescope will also have the advantage of having a completely new electronics which could reach a sampling frequency of up to $2 \mathrm{GHz}$, i.e. a timing resolution of 0.5 ns. The arrival times of the photons on the pixels add information about the structure of the shower that can be used in a more sophisticated reconstruction procedure. In particular, the pixel times could help to evaluate the impact parameter of the event. A reconstruction strategy using the pixel timing is under development.

\section{ANALYSIS CUTS FOR THE MONO REGIME}

Hadron rejection cut. The Hillas parameters toghether with other robust parameters of the event can be used for the definition of a hadron rejection cut. The optimisation of the background rejection cut for the pure T5 Mono events has been performed with the Fisher algorithm implemented in the multi-variate analysis package TMVA [4]. We choose to train the algorithm with the nominal distance, the Hillas length and width, and the total cleaned charge in the event. The Fisher response to the sample of simulated gammas and protons is shown in Fig. 1. For this study we chose to use a cut at a value 


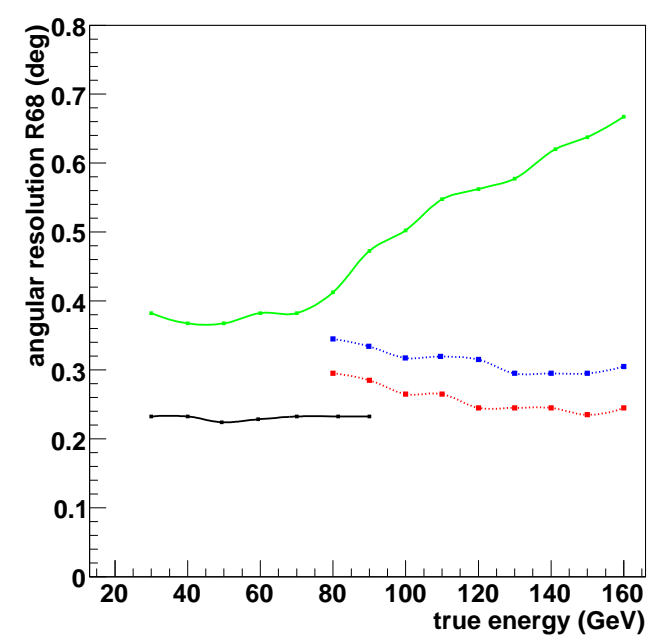

FIGURE 2. Angular resolution for the different HESS-II detection regimes: the upper continuous line shows the curve for the pure T5 Mono events after image filtering, while the lower continuous line shows the curve after the background rejection cut (cut 1) and the nominal distance cut (cut 2). The remaining curves are obtained in the case of the semi-Stereo plus full-Stereo events at filtering and reconstruction level (the upper dotted line is for the Hillas case while the lower dotted line is for the Model3D case) and no analysis cuts were applied; the final stereo angular resolution is expected to be much better.

of 0.1 (called cut 1 ), where the maximum quality factor $(S / \sqrt{B}$, being $S$ the signal and $B$ the background) is reached.

Image shape cut. An additional cut on the nominal distance (cut 2) is required in order to reject the events giving images at the border of the camera, and to reject the events hitting the telescope too close to the source direction, which give non-elliptical images. We save only the events giving images at a nominal distance greater than $>0.45^{\circ}$ and smaller than $<1^{\circ}$ for this case where the source is at the camera centre.

Cut on the angular resolution. The final cut (cut 3 ) is a selection of the well-reconstructed events based on the angular resolution, which will be introduced in Sec. 5. A summary of the analysis cuts for the pure Mono regime is given in Tab. 2 .

\section{ANGULAR RESOLUTION AND EFFECTIVE AREA}

With the events passing the above-mentioned cuts 1 and 2, we estimated the expected angular resolution and effective area for the pure Mono regime.

Angular resolution. The angular resolution, defined as
$68 \%$ containment radius for all the pure T5 Mono events after image filtering and reconstruction, is shown in Fig. 2 with the upper continuous line, while the resulting curve after the background rejection cut and the nominal distance cut is shown with the lower continuous line. We also estimated the angular resolution of the hybrid and stereo events after reconstruction with the Hillas (upper dotted line) and the Model3D (lower dotted line) algorithms without any cuts, for comparison. The angular resolution for the pure $\mathrm{T} 5$ events after cuts $(1+2)$ is of the order of $0.25^{\circ}$.

Effective area. For the calculation of the effective area, another quality cut on the squared angular deviation between the source position and the reconstructed direction $\theta^{2}$ (cut 3 ) is applied: we select all the events having a $\theta^{2}<0.13 \mathrm{deg}^{2}$. The effective area resulting after the three cuts given in Tab. 2 is shown in Fig. 3. The area in the T5 Mono range after cuts is shown with the lower continuous curve: it has a maximum around $40 \mathrm{GeV}$ then it drops dramatically at about $80 \mathrm{GeV}$ where the semi-Stereo and full-Stereo detection regimes take over. In the upper dotted and lower dotted lines we show the curves for the Hillas and Model3D (pre-cuts).

\section{SHOWER ENERGY EVALUATION}

A dedicated study for the evaluation of the energy of the pure T5 events has been carried out using a NN approach: we used the module called MultiLayerPerceptron developed in the ROOT framework [5]. We have trained our NN with the events passing the three cuts summarised in Tab. 2 and with the same parameters as used for the multivariate analysis described in Sec. 4. The architecture of our network (see Fig. 4) is given by 4 input nodes, 2 hidden layers composed by 4 nodes each and one output node $(4: 4: 4: 1)$. The resulting energy resolution defined as $\Delta E / E$ is shown in Fig. 5 in the upper continuous line, while the bias in the evaluation of the energy defined as $\langle E\rangle / E$ is shown in lower continuous line. The two curves are both obtained in the case of the overtraining check. The bias in the evaluation of the energy was found to be essentially due to the events having a large impact parameter: these events globally tend to lower the energy reconstruction performance. The dotted lines on Fig. 5 represent the energy resolution and the bias for the events having an impact parameter smaller than about $130 \mathrm{~m}$.

\section{CONCLUSIONS}

From our preliminary study of the performance of the HESS-II telescope we found the angular resolution for the pure $\mathrm{T} 5$ events to be of the order of $0.25^{\circ}$ in the $[30$ 
TABLE 2. Summary of the quality cuts applied for the pure T5 analysis. Cut 1 has been optimised for background rejection, cut 2 to reject the incomplete or round images, cut 3 to select the well recontructed events.

\begin{tabular}{cc}
\hline Cut number & Value \\
\hline Cut 1 & Fisher response $>0.1$ \\
Cut 2 & $0.45^{\circ}<$ nominal distance $<1^{\circ}$ \\
Cut 3 & $\theta^{2}<0.13 \mathrm{deg}^{2}$ \\
\hline
\end{tabular}

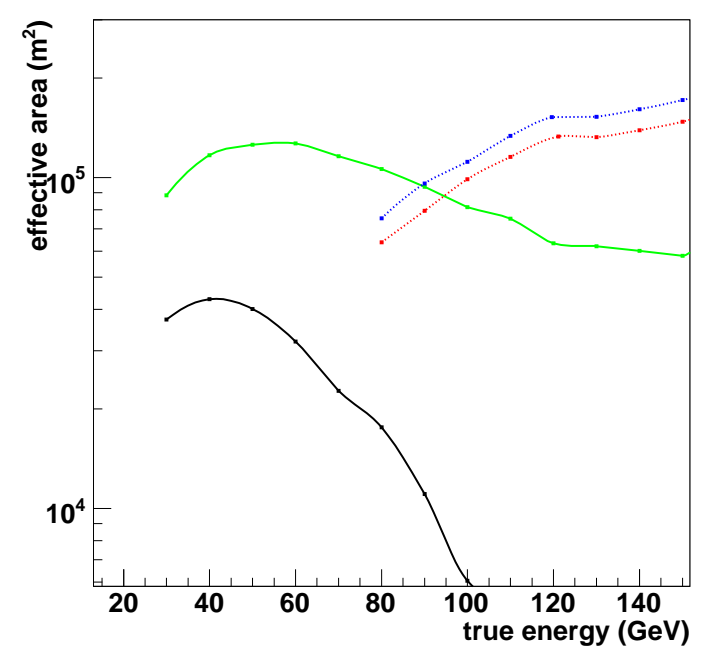

FIGURE 3. Effective area given in $\mathrm{m}^{2}$ for the different HESS-II detection regimes: at filter level (upper continuous line) and after cuts (lower continuous line) for the pure T5 events; at filter level for the semi-Stereo and full-Stereo events for two different reconstruction algorithms, the upper dotted line represents the results obtained with the Hillas algorithm, while the lower dotted line represents the Model3D resulting curve, see text.

$\mathrm{GeV}, 90 \mathrm{GeV}$ ] energy range after the hadron rejection and image shape cuts. The effective area after the additional cut on the angular resolution is found to be $4 \times 10^{4} \mathrm{~m}^{2}$ at the maximum value of $40 \mathrm{GeV}$. Our current evaluation of the energy resolution varies from $40 \%$ to $20 \%$ as a function of the energy, while the bias spans from $+40 \%$ to $-40 \%$ : a more detailed study is needed in order to suppress this effect. At the time of writing we already know that we can improve the overall performance of the analysis, by adding the timing information and by further optimising the neural network layers and nodes. The results we have obtained for this pure T5 Mono analysis can be considered as a pessimistic case or a preliminary version of the final analysis.

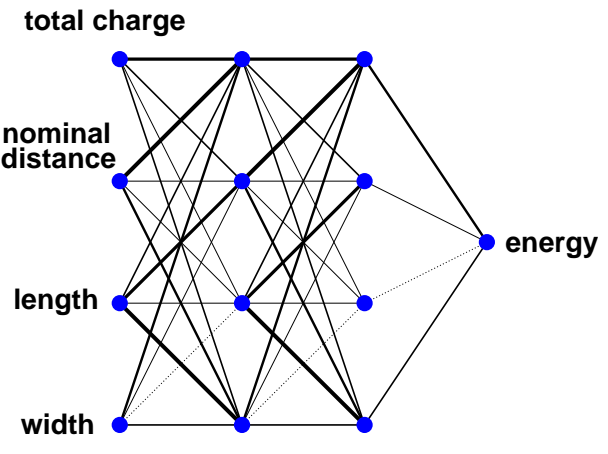

FIGURE 4. Neural network used for the evaluation of the energy for the pure Mono events seen only by T5. It consists of 4 input nodes, 2 hidden layers holding 4 nodes each and one output node.

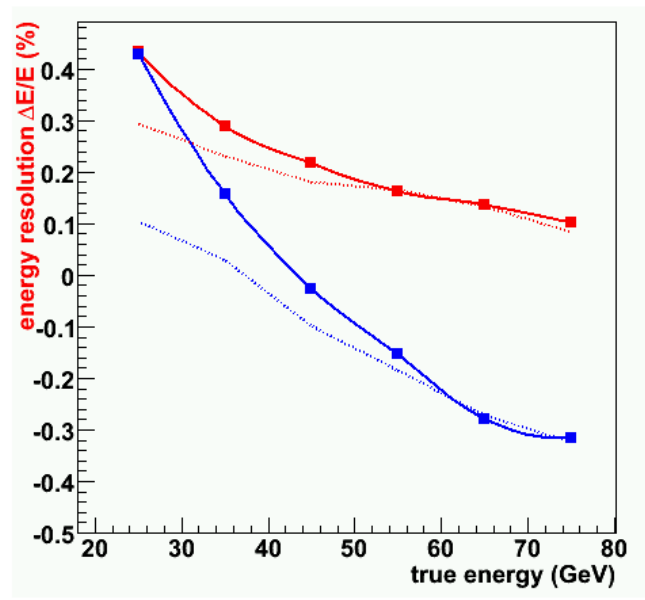

FIGURE 5. Energy resolution (upper continuous line) and bias curve (lower continuous line) obtained with a neural network (see text) in the case of the pure T5 Mono events. The dotted curves represent the resolution and the bias for the events having an impact parameter smaller than $130 \mathrm{~m}$.

\section{REFERENCES}

1. C. Deil et al. for the H.E.S.S. Collaboration, H.E.S.S. II - Telescope Structure, Mirror and Drive System

2. W. Hofmann, I. Jung, A. Konopelko, et al. 1999, Astropart. Phys., 12, 135

3. M. Lemoine-Goumard, B. Degrange, M. Tluczykont, Astropart. Phys. 25 (2006) 195-211

4. http://tmva.sourceforge.net/

5. http://root.cern.ch/ Neural network classes 